\title{
Response to Regarding Microbiological Control of Cellular Products: The Relevance of the Cellular Matrix, Incubation Temperature, and Atmosphere for the Detection Performance of Automated Culture Systems. Transfus Med Hemother. 2020; 47:254-63
}

\author{
Frank Günther ${ }^{a, b}$ \\ ${ }^{a}$ Medical Microbiology and Hygiene, Department of Infectious Diseases, Heidelberg, Germany; ${ }^{b}$ Division of \\ Infection Control, Medical Microbiology and Hygiene, Philipps University of Marburg, Marburg, Germany
}

We appreciate the interest in our article "Microbiological Control of Cellular Products: The Relevance of the Cellular Matrix, Incubation Temperature, and Atmosphere for the Detection Performance of Automated Culture Systems" [1] and your comments. The primary goal of the mentioned study was to delineate the significance of various influencing factors on the detection performance of microbiological contaminants by cell metabolism or carbon dioxide-based detection systems. Therefore, we did not describe the general suitability of this system or the different available culture media for the approval testing of specific stem cell preparations.

However, Prof. Moog raises an important point: depending on the conditions of collection and processing of the cellular product it might be helpful using culture bottles with neutralizing absorber resins to safely detect microbial contaminations, especially in presence of antimicrobial substances.

As commented, we used different cell concentrations of easily available PBMCs in order to point out the influence of cell metabolism or carbon dioxide production of cells contained in the matrix on the detection properties of the culture system. In fact, it was not the aim of our study to simulate the conditions when testing specific stem cell preparations. Since we focused on the influence factor of cell metabolism and atmospheric conditions, no specific cell concentration or composition of the functional cell population was aimed in our study.

We agree to the comment that the presence of different functional leukocyte populations can be of considerable relevance in microbiological control. However, this must then be considered in the context of the product-specific validation process.

\footnotetext{
References $\quad 1$ Günther SK, Geiss C, Kaiser SJ, Mutters NT, Günther F. Microbiological Control of Cellular Products: The Relevance of the Cellular Matrix, Incubation Temperature, and Atmosphere for the Detection Performance of Automated Culture Systems. Transfus Med Hemother. 2020 Jun;47(3):254-63.
} 